\title{
RAZONAMIENTO PEDAGÓGICO DE DOCENTES DE LICENCIATURA EN CULTURA FÍSICA, RECREACIÓN Y DEPORTE EN INVESTIGACIÓN FORMATIVA
}

\author{
THE PEDAGOGICAL REASONING OF TEACHERS OF LICENCIATURA EN CULTURA FÍSICA, \\ RECREACIÓN Y DEPORTE IN EDUCATIONAL RESEARCH
}

Carlos Alberto Salazar Díaz ${ }^{1}$

\section{Resumen}

\begin{abstract}
En este artículo se presentan los resultados parciales de una investigación más amplia titulada: "Conocimiento pedagógico de investigación formativa en tres programas de pregrado de la Universidad del Atlántico". El propósito del presente estudio es elaborar un perfil de interacción pedagógica de los docentes de cultura física, recreación y deporte, a partir de los comportamientos observables en el aula que permitan inferir los procesos de razonamiento pedagógico. La técnica de recolección de datos fue la observación no participante. El instrumento principal de recolección de datos que se implementó fue una rúbrica de observación de interacciones pedagógicas. Los resultados muestran diferencias entre los modos de interacción y pensamiento de docentes expertos y docentes en formación. La discusión involucra los planteamientos de investigaciones pioneras en el uso de las categorías de pensamiento pedagógico. Las conclusiones señalan los beneficios de indagar el razonamiento pedagógico de docentes expertos y docentes en formación.
\end{abstract}

Palabras claves: razonamiento pedagógico, investigación formativa, cultura física, recreación y deporte.

\section{Abstract}

This paper reports partial results of a broader research entitled: Pedagogical Content Knowledge of Formative Research in three undergraduate programs at the Universidad del Atlántico. The purpose of this study is to develop a profile of pedagogical interaction of teachers from observable behaviors in the classroom that allow infer what pedagogical reasoning processes. The data collection techniques were non-participant observation. The main instrument of data collection is an observation rubric of pedagogical interactions. Results reveal differences between expert teachers and student teachers modes of interaction and thinking. The discussion involves pioneered researches of using categories of pedagogical thought.The findings point to the benefits of investigating expert teachers and student teachers Pedagogical Reasoning.

Keywords: pedagogical reasoning, formative research, physical culture, sport and recreation.

Fecha de recepción: 15 de mayo de 2015

Fecha de aprobación: 10 de octubre de 2015

Para citar este artículo:

Salazar, C.A. (2015). Razonamiento pedagógico de docentes de Licenciatura en Cultura Física,

Recreación y Deporte en investigación formativa. Lúdica Pedagógica, (22), 111-121.

1 Magíster en Educación del SUE-Caribe, licenciado en Humanidades y Lengua Castellana, sede Universidad del Atlántico. Docente catedrático con dedicación de medio tiempo, adscrito a la Facultad de Ciencias de la Educación de la misma institución. Miembro activo del Grupo Educativo de Ciencia y Tecnología (GECIT), reconocido en categoría A por Colciencias. Correo electrónico: csalazardiaz@mail.uniatlantico.edu.co 


\section{INTRODUCCIÓN}

Parte esencial del reconocimiento de una institución de educación superior se refiere al desempeño acreditado en campos generales de investigación (humanística, artística, tecnológica, etc.), y en áreas específicas de investigación científica. Lo anterior está contemplado en la Ley 30 de 1992, concretamente en el artículo 19, que resalta la importancia de actividades como "la investigación científica o tecnológica; la formación académica en profesiones o disciplinas y la producción, desarrollo y transmisión del conocimiento y de la cultura universal y nacional". Desde entonces la investigación ha sido considerada como un aspecto clave en los procesos de acreditación de las universidades; en consecuencia, se han promovido medidas para su fortalecimiento y regulación en las universidades (Anzola, 2005).

Justamente, el concepto de investigación formativa (IF) surge desde una perspectiva de la universidad como fuente de producción y comunicación del conocimiento científico. Por ende, el perfil profesional de estudiantes y docentes de diferentes áreas del conocimiento concedió espacio a actitudes y competencias investigativas (Lara, 2006; De la Ossa, Pérez, Patiño y Montes, 2012; Aldana, 2012). Más allá, la IF ha sido empleada tanto para la actualización y reflexión sobre la docencia, como en la extensión y proyección social de la universidad (Sancho, 2001; López, 2011).

En cuanto a los fundamentos pedagógicos y didácticos de la IF, existen múltiples tipos de aprendizaje compatibles, entre ellos: el basado en problemas, el autodirigido, el fundamentado en la indagación y el colaborativo, etc. En todo caso, son destacables las estrategias didácticas que Álvarez (2013) propone desde la pedagogía socrática, dado que integra la enseñanza por proyectos, los ensayos de carácter argumentativo, el seminario alemán, entre otros. En el fondo, esta autora resalta que más allá de la apropiación de técnicas y metodologías investigativas, los docentes deben enseñar ética, valores ciudadanos y pensamiento crítico, y a partir de allí comenzar la construcción de saberes útiles para el desarrollo personal y social de cara a la transformación de la realidad.

\section{EL RAZONAMIENTO PEDAGÓGICO EN INVESTIGACIÓN FORMATIVA}

Los profesores deben aprender a utilizar su base de conocimiento de la enseñanza para proporcionar fundamentos a las elecciones y acciones pedagógicas. En consecuencia, la formación docente debe trabajar con las creencias que guían las acciones del profesor, con los principios y pruebas que sustentan las decisiones que hacen los docentes. Por todo lo anterior, Shulman (1987) propone un modelo de razonamiento y acción pedagógica que sucede idealmente en un ciclo de procesos, y que parte y termina en la (nueva) comprensión:

\section{Comprensión}

De propósitos, estructuras de la materia de estudio, las ideas dentro y fuera de la disciplina.

\section{Transformación}

Preparación: interpretación crítica y análisis de textos, estructuración y segmentación, desarrollo de un repertorio curricular, y la clarificación de propósitos.

Representación: el uso de un repertorio representacional que incluye analogías, metáforas, ejemplos, demostraciones, explicaciones, y así sucesivamente.

Selección: elección entre un repertorio de instrucción que incluye modos de enseñanza, organización, gestión y organización.

Adaptación y preparación a las características del estudiante: consideración de conceptos, ideas preconcebidas, concepciones erradas y dificultades, idioma, cultura y motivaciones, clase social, género, edad, habilidad, aptitud, intereses, autoconceptos, y atención.

\section{Instrucción}

Gestión, presentaciones, interacciones, trabajo en grupo, disciplina, humor, cuestionamiento, y otros aspectos de la enseñanza activa, enseñanza por descubrimiento o indagación, y formas observables de la enseñanza en el aula.

\section{Evaluación}

Comprobación de la comprensión del estudiante durante la enseñanza interactiva.

Prueba de comprensión de los estudiantes al final de las lecciones o unidades.

Evaluación del desempeño propio, y ajuste por experiencias.

\section{Reflexión}

Revisión, reconstrucción, recreación y analizar críticamente las actuaciones propias de la clase, y explicaciones fundamentadas en la evidencia. 
Nueva comprensión

De los propósitos, materia de estudio, estudiantes, enseñanza, y misma persona.

Consolidación de nuevos entendimientos y aprendizajes desde la experiencia. (Shulman, 1987, p. 101).

Desde la perspectiva de Shulman, el proceso que lleva a un docente novato a convertirse en un docente experto implica una compleja interacción entre diversos conocimientos, acciones y pensamientos, que se han consolidado a través de experiencias y oportunidades de enseñanza. Visto así, a lo largo de la carrera docente existen hitos que marcan avances y retrocesos en el razonamiento y la acción pedagógica, que deben ser explorados y documentados sistemáticamente.

En efecto, Shulman (1986) cuestionó la tradición y los alegatos sobre el conocimiento y las habilidades necesarias para ejercer la docencia, con la finalidad de resolver preguntas como: ¿Qué necesitan saber los profesores? y ¿Cuáles son las fuentes del conocimiento de los docentes? Las respuestas a estas preguntas estarían enmarcadas en la base de conocimiento de la enseñanza, que va más allá de la caracterización de las habilidades de enseñanza a partir de la investigación, y trasciende hasta la sabiduría de la práctica; es decir, el conocimiento estratégico y la compresión de los procesos y contenidos que inciden en la docencia. Fue así como Shulman (1987) propuso siete categorías de la base de conocimiento de los docentes:

1. Conocimiento del contenido: se refiere a la cantidad y organización del conocimiento per se en la mente del profesor. Además, requiere comprender la estructura de los materiales de estudio, e ir más allá del conocimiento de hechos y conceptos de un campo disciplinar.

2. Conocimiento pedagógico general: se refiere especialmente a los principios generales y las estrategias de gestión del aula y de la organización de ambientes de aprendizaje.

3. Conocimiento del currículo: también llamado conocimiento curricular, se refiere a programas diseñados para la enseñanza de materias y temas particulares en un determinado nivel, y a materiales didácticos disponibles en relación con esos programas. Por una parte, hace alusión a la capacidad del profesor para relacionar el contenido de un determinado curso o lección, a temas o cuestiones discutidas simultáneamente en otras clases. Por otra parte, implica la familiaridad con los temas y asuntos abordados en la misma área durante los años anteriores y posteriores, y los materiales que los representan.

4. Conocimiento de los fines educativos, propósitos, y valores, y sus fundamentos históricos y filosóficos: se refiere a la necesidad que tiene el docente para tomar parte en su comunidad académica, que claramente está situada en el espacio y el tiempo, que además responde tanto a una tradición epistémica, axiológica y normativa, como a conjuntos de acuerdos y metas institucionales reconocidas.

5. Conocimiento de los aprendices y sus características: se refiere a las creencias, motivaciones y características psicológicas, de los estudiantes, que hacen posible la selección pertinente y oportuna de actividades y oportunidades específicas para el aprendizaje. Este conocimiento sirve para orientar a los docentes en los roles y los modos de interacción que asumirán en el aula. Aunque en última instancia, el aprendizaje sigue siendo responsabilidad de los estudiantes.

6. Conocimiento del contexto educativo: va desde el funcionamiento del grupo o clase, la gestión directiva y financiera, hasta el carácter de las familias, comunidades y culturas que se vinculan del establecimiento educativo.

7. Conocimiento pedagógico del contenido (CPC): se refiere a la enseñabilidad del conocimiento del contenido, e incluye comprensión sobre dificultades u oportunidades en el aprendizaje de temas específicos. Asimismo, conceptos y preconceptos de los estudiantes, que se corresponde con un reportorio de ideas y acciones que va desde estrategias de enseñanza, investigación, hasta las formas de representar y comunicar las temáticas de la asignatura (pp. 92-93). 
En efecto, el CPC representa la combinación de contenido y pedagogía en la comprensión de cómo se organizan temas particulares, para ser representados y adaptados a los diversos intereses y capacidades de los estudiantes. En últimas, el CPC es la categoría más factible para distinguir la comprensión del especialista en la temática, de la comprensión del pedagogo en la enseñanza.

En orden a retratar el razonamiento pedagógico propio de docentes expertos en la asignatura de IF, fue necesario observar, sistematizar, analizar e interpretar los comportamientos tanto de estudiantes como docentes, que permitieran consolidar un repertorio interacciones referentes a la asignatura. Para tal fin, se implementó una rúbrica de observación que emplea categorías del conocimiento pedagógico de docentes en formación y docentes expertos, propuestas inicialmente por Gatbonton (1999, 2008), y ampliadas por Mullock (2006). Estos investigadores demostraron que es posible acceder a patrones de conocimiento sobre la enseñanza y el aprendizaje que los docentes de una misma asignatura, con diferentes niveles de formación y experiencia, demuestran en sus comportamientos durante clases.

Tabla 1. Aspectos de razonamiento pedagógico y de categorías de interacción pedagógica

\begin{tabular}{|c|c|c|}
\hline \multicolumn{2}{|c|}{$\begin{array}{l}\text { Razonamiento pedagógico } \\
\text { (Shulman, 1987) }\end{array}$} & \multirow{2}{*}{$\begin{array}{c}\text { Categorías (Gatbonton 1999, 2008; } \\
\text { Mullock, 2006) }\end{array}$} \\
\hline Proceso & Subproceso & \\
\hline Comprensión & & $\begin{array}{c}\text { Contenidos } \\
\text { Capacidad de Comprensión }\end{array}$ \\
\hline \multirow{4}{*}{ Transformación } & Preparación & $\begin{array}{l}\text { Manejo del lenguaje } \\
\text { Planificación }\end{array}$ \\
\hline & Representación & Sugerencias y ejemplos \\
\hline & Selección & Decisiones \\
\hline & Adaptación y preparación & $\begin{array}{l}\text { Habla espontánea } \\
\text { Creencias } \\
\text { Afectos }\end{array}$ \\
\hline Instrucción & & $\begin{array}{c}\text { Nota de comportamiento } \\
\text { Control del tiempo } \\
\text { Trabajo de grupo/pares } \\
\text { Lista de nombres }\end{array}$ \\
\hline Evaluación & & $\begin{array}{l}\text { Comprobación de nivel } \\
\text { Comprobación de procedimiento } \\
\text { Conocimiento de los estudiantes }\end{array}$ \\
\hline Reflexión & & $\begin{array}{l}\text { Verificación de problemas } \\
\text { Experiencias pasadas } \\
\text { Conocimiento investigado }\end{array}$ \\
\hline Nueva comprensión & & $\begin{array}{c}\text { Autocrítica } \\
\text { Repaso de progresos }\end{array}$ \\
\hline
\end{tabular}

Fuente: elaboración propia. 


\section{METOdOLOGÍA}

El enfoque cualitativo fue asumido en la presente investigación, puesto que "los investigadores cualitativos estudian las cosas en sus escenarios naturales, tratando de entender o interpretar los fenómenos en función de los significados que las personas les dan" (Denzin y Lincoln, 2012, p. 48). Concretamente, la investigación adoptó el diseño del estudio de caso colectivo (o múltiple), puesto que es "un proyecto que trata de abordar un problema en cuestión, al tiempo que incorpora la literatura base que ayuda a conceptualizar una teoría" (Hancock y Algozzine, 2006, p. 85). Además, este tipo de estudios que implican una descripción exacta y profunda de una o varias personas, dado que interesa la subjetividad, la finalidad es evidenciar comportamientos excepcionales (Marczyk, DeMatteo y Festinger, 2005).

\section{Técnicas e instrumentos de recolección de datos}

Se empleó la observación no participante, con respaldo en la rúbrica de observación de interacciones pedagógicas; está por constituida 21 categorías para estimar la frecuencia de los comportamientos de docentes y estudiantes durante las clases (Gatbonton 1999, 2008; Mullock, 2006).

\section{Marco contextual}

El escenario de investigación fue la Universidad del Atlántico, en Barranquilla. En particular, se decidió indagar el razonamiento pedagógico de docentes de las asignaturas de Investigación Formativa I y III, en la Licenciatura de Cultura Física, Recreación y Deportes.

\section{Muestreo y muestra}

Se tomó una muestra intencionada, también llamada por conveniencia (Hérnandez, Fernández y Baptista, 2010, p. 393), que consiste en seleccionar casos que ofrezcan información abundante para un estudio detallado, sin necesidad de generalizaciones sobre cada caso particular (McMillan y Schumacher, 2005); no obstante, los resultados guardan un margen de transferencia a muestras similares en tiempo y lugar.

\section{Sujetos participantes}

En el programa de Licenciatura en Recreación, Cultura Física y Deporte se observaron y registraron en videograbación las clases del docente 1, quien estaba a cargo de Investigación Formativa I; y las clases del docente 2, a cargo de Investigación Formativa III. En total fueron ana- lizadas seis clases con duración promedio de 81 minutos. Ambos profesores de género masculino, con una edad promedio de 52 años. La experiencia de enseñanza de los docentes es de 19 años, a nivel de educación superior. Todos con estudios de posgrado concluidos, uno a nivel de maestría, el otro a nivel de doctorado.

\section{RESULTADOS}

\section{Modos de interacción pedagógica del docente 1}

La autocrítica enmarca las formas de interacción pedagógica que prevalecen en las clases de docente 1 (tabla 2), muestra de ellos son, en su orden, la identificación de mejoras posibles en la práctica pedagógica, las sugerencias ante las dificultades de un tema de estudio, y la promoción de la autoevaluación en la clase. Otra forma importante en la manera de interactuar de este docente es la verificación de problemas, lo cual conlleva a la revisión de errores recurrentes en una tarea y a la comprobación de dificultades de apropiación conceptual. También, el habla espontánea es un modo de interacción pedagógica que es notorio cuando el docente da orden de silencio o moderación del volumen de la voz. 0 bien cuando hace llamados de atención ante interferencias, e inclusive sanciona las divagaciones sobre la temática de la clase.

Más aún, el docente 1 demuestra su autoridad cuando interactúa en su práctica pedagógica, puesto que toma las decisiones, es decir, es quien hace la asignación de temáticas de investigación, la asignación de roles y funciones para próximas clases, y orienta el consenso de compromisos para la próxima clase. De la misma manera, la comprobación de procedimientos implica importantes interacciones pedagógicas para este docente, por ejemplo: la repetición de las instrucciones de trabajo, la comprobación de la atención y escucha activa, la confirmación de resultados al final de un procedimiento, y el aseguramiento de la claridad y sencillez de enunciados cuando participan sus estudiantes.

\section{Modos de interacción pedagógica de los estudiantes} a cargo del docente 1

Los estudiantes participan de manera activa y constante en las clases del docente 1 , tanto que sus interacciones pedagógicas evidenciadas en su comportamiento superan las interacciones propias del profesor (tabla 2). Siendo así, el habla espontánea es el modo de inte- 
racción pedagógica predominante, evidenciable en las participaciones voluntaria y espontánea para expresar puntos de vista y opiniones, así como la promoción del respecto por los turnos de habla. Otra forma de interactuar importante para los docentes en formación es la autocrítica, que se manifiesta mediante el reconocimiento de limitaciones en la práctica pedagógica.

Por consiguiente, estos docentes en formación hacen cuestionamientos a las respuestas de los estudiantes, es decir que entre los mismos compañeros de estudio se abren espacios para la formulación de preguntas sobre el tema visto en clase; así, se confirma que las interacciones en el aula demuestran el conocimiento de los estudiantes. En consecuencia, futuros licenciados en cultura física, recreación y deporte muestran alto interés en los contenidos de la investigación formativa, por eso tienden a dar explicaciones sobre un tema de clase mediante la argumentación y demostración. En este mismo sentido, los estudiantes tienden a demostrar conocimiento investigado a través de la socialización de conocimientos indagados y el aporte de evidencias de acceso y permanencia en el escenario de investigación.

\section{Perfil de razonamiento pedagógico del docente 1}

El predominio de categorías de interacción pedagógica como la autocrítica y el repaso de progresos señala que el docente está enfocado en los procesos asociados a la nueva comprensión (tabla 1). Lo anterior implica que el docente 1 emplea estrategias específicas para la documentación, análisis y discusión con los docentes en formación, con miras a indagar la cotidianidad de los actos pedagógicos en el aula y logar una aprendizaje experiencial trascedente (Shulman, 1987). De igual forma, el proceso de reflexión es importante para este docente, debido a que la verificación de problemas y la confirmación del conocimiento investigado son categorías evidentes en su interacción pedagógica.

Tabla 2. Frecuencia de interacciones pedagógicas: docente 1.

\begin{tabular}{|c|c|c|c|c|}
\hline & \multicolumn{4}{|c|}{ Interacciones pedagógicas } \\
\hline & $\begin{array}{c}\text { Frec. } \\
\text { Est. }\end{array}$ & $\begin{array}{c}\text { Frec. } \\
\text { Doc } 1 .\end{array}$ & $\begin{array}{l}\text { Frec. } \\
\text { Total } \\
\end{array}$ & $\begin{array}{c}\text { Pct. } \\
\text { Caso (\%) }\end{array}$ \\
\hline 1. Manejo del lenguaje & 12 & 7 & 19 & 4,4 \\
\hline 2. Conocimiento de los estudiantes & 27 & 3 & 30 & 6,9 \\
\hline 3. Nota de comportamiento & 12 & 10 & 22 & 5,1 \\
\hline 4. Decisiones & 1 & 12 & 13 & 3 \\
\hline 5. Repaso de progresos & 4 & 10 & 14 & 3,2 \\
\hline 6. Comprobación de procedimiento & 5 & 11 & 16 & 3,7 \\
\hline 7. Creencias & 3 & 2 & 5 & 1,1 \\
\hline 8. Afectos & 0 & 1 & 1 & 0,2 \\
\hline 9. Autocrítica & 38 & 32 & 70 & 16,2 \\
\hline 10. Contenidos & 20 & 14 & 34 & 7,9 \\
\hline 11. Control del tiempo & 8 & 1 & 9 & 2,1 \\
\hline 12. Verificación de problemas & 15 & 19 & 34 & 7,9 \\
\hline 13. Experiencias pasadas & 15 & 9 & 24 & 5,5 \\
\hline 14. Planificación & 3 & 5 & 8 & 1,8 \\
\hline 15. Trabajo de grupo/pares & 11 & 1 & 12 & 2,8 \\
\hline 16. Lista de nombres & 0 & 9 & 9 & 2,1 \\
\hline
\end{tabular}




\begin{tabular}{|l|c|c|c|}
\hline 17. Capacidad de comprensión & 0 & 0 & 0 \\
\hline 18. Conocimiento investigado & 19 & 11 & 30 \\
\hline 19. Comprobación de nivel & 0 & 0 & 0 \\
\hline 20. Habla espontánea & 49 & 15 & 64 \\
\hline 21. Sugerencias y ejemplos & 6 & 10 & 16,8 \\
\hline Total de interacciones & 248 & 182 & 430 \\
\hline Porcentaje de interacciones & $57.7 \%$ & $42.3 \%$ & 430 \\
\hline
\end{tabular}

Así, el docente 1 promueve el aprendizaje que surge de la experiencia, a partir de análisis sistemático que permita ver en retrospectiva las prácticas pedagógicas que fueron indagadas junto a sus estudiantes de licenciatura. Además, los procesos de transformación que a su vez enmarcan subprocesos como la representación, la selección y adaptación y preparación, están asociados a categorías de interacción pedagógica como las sugerencias y ejemplos, las decisiones y el habla espontánea.

Por ende, el docente 1 motiva y orienta a sus estudiantes, analiza de ideas principales contenidas en textos producidos tanto por ellos mismos como por autores reconocidos. Además, este profesor hace explícito un enfoque pedagógico concreto, desde el cual emplea estrategias de enseñanza convencionales como diálogos socráticos, oportunidades de repetición del trabajo de los estudiantes, y promoción del aprendizaje mediante reflexiones pedagógicas y didácticas sobre las clases de docentes en otras asignaturas, por ejemplo, Fisiología del Ejercicio, Artes Marciales I, Gimnasia I, etc.

\section{Modos de interacción pedagógica del docente 2}

La interacción pedagógica del docente 2 gira en torno a los contenidos, ya que son constantes y detalladas las acciones dirigidas a la presentación de estructura y contenidos de la asignatura, la presentación de un tema nuevo, y la comunicación de propósitos de la actividad en clase. También, en clases es recurrente el manejo de diversas fuentes de información, la socialización de conocimientos indagados, y los conceptos previos lluvia de ideas aportadas en clase, esto es prueba de que el conocimiento investigado es otra categoría de interacción pedagógica. Por consiguiente, el profesor se ocupa con frecuencia de dar explicaciones alternativas, y en solicitar alternativas de explicación a los estudiantes, sin dejar de lado que ofrece aclaraciones generales y aclaraciones particulares sobre metodología de la investigación. De donde resulta que, la verificación de problemas se facilite mediante la comprobación de dificultades de apropiación conceptual, la detección de expresión insegura de una idea, y la manifestación de falta de claridad conceptual de los futuros licenciados en cultura física, recreación y deporte.

Asimismo, para el docente 2 es importante la autocrítica que hace posible tanto el señalamiento de circunstancias adversas en la clase, como la presentación de sugerencias ante las dificultades de un tema de estudio. Lo anterior da lugar al repaso de progresos mediante resolución dudas o vacíos conceptuales sobre la teoría y la práctica, y el repaso de conceptos, términos o palabras clave. Por último, es evidente que el docente toma las decisiones de la clase, puesto que se encarga de la asignación de temáticas de investigación y la realización de actividades en el aula, como exposiciones, mesa redonda, elaboración de mapas conceptuales, etc.

\section{Modos de interacción pedagógica de} los estudiantes a cargo del docente 2

Los estudiantes tienen dominio de los contenidos porque demuestran una clara delimitación conceptual de la materia de estudio, incluso cuando son capaces de dar explicación de un tema de clase mediante argumentación y demostración (tabla 3). En consecuencia, la interacción pedagógica de los estudiantes se concreta en el aporte de conceptos y lluvia de ideas en clase, así como muestras de evidencias escritas de lectura comprensiva y crítica y la resolución de problemas relacionados con el tema de clase; de esta manera los docentes en formación evidencian la adquisición de un conocimiento investigado. 
La autocrítica, también, es importante para los estudiantes de Licenciatura en Cultura Física, Recreación y Deporte, lo cual se manifiesta en interacciones pedagógicas referidas a la identificación de mejoras posibles en la práctica pedagógica, el reconocimiento de limitaciones en la práctica pedagógica y, sobre todo, en el señalamiento de circunstancias adversas en la clase. Además, el conocimiento de los estudiantes es notable cuando estos contribuyen activamente a la formulación de preguntas sobre el tema visto en clase, lo cual facilita la valoración del desempeño grupal o individual.

También, el habla espontánea, en general, y las participaciones voluntaria y espontánea, en particular, contribuyen al dinamismo de las interacciones pedagógicas. Sumado a lo anterior, las sugerencias y ejemplos destacan la habilidad para lograr la contextualización de los enunciados derivados de lecturas de material bibliográfico, y pone en relieve la capacidad de repetición o paráfrasis de una idea principal que a su vez contribuye a la construcción colectiva del conocimiento en la asignatura.

\section{Perfil de razonamiento pedagógico del docente 2}

El proceso de comprensión es el aspecto del razonamiento pedagógico que se destaca en el comportamiento del docente 2 . Siendo así, el profesor comprende críticamente el conjunto de ideas y conceptos que va a enseñar, por eso confronta diversos autores y hace triangulación de teorías sobre una determinada temática. Luego, la reflexión es otro proceso importante, ya que este docente examina detenidamente la verificación de problemas y el conocimiento investigado, a propósito de cotejar las metas de enseñanza alcanzadas con las expectativas de desempeño proyectadas (tabla 3 ).

Tabla 3. Frecuencia de interacciones pedagógicas: docente 2

\begin{tabular}{|c|c|c|c|c|}
\hline & \multicolumn{4}{|c|}{ Interacciones pedagógicas } \\
\hline & $\begin{array}{c}\text { Frec. } \\
\text { Est. }\end{array}$ & $\begin{array}{l}\text { Frec. } \\
\text { Doc. }\end{array}$ & Frec. Total & $\begin{array}{c}\text { Pct. } \\
\text { Caso (\%) }\end{array}$ \\
\hline 1. Manejo del lenguaje & 0 & 1 & 1 & 3 \\
\hline 2. Conocimiento de los estudiantes & 32 & 3 & 35 & 11,4 \\
\hline 3. Nota de comportamiento & 2 & 6 & 8 & 2,6 \\
\hline 4. Decisiones & 1 & 8 & 9 & 2,9 \\
\hline 5. Repaso de progresos & 3 & 6 & 9 & 2,9 \\
\hline 6. Comprobación de procedimiento & 1 & 1 & 2 & 0,6 \\
\hline 7. Creencias & 1 & 1 & 2 & 0,6 \\
\hline 8. Afectos & 0 & 0 & 0 & 0 \\
\hline 9. Autocrítica & 34 & 8 & 42 & 13,6 \\
\hline 10. Contenidos & 43 & 25 & 68 & 22,1 \\
\hline 11. Control del tiempo & 0 & 0 & 0 & 0 \\
\hline 12. Verificación de problemas & 4 & 12 & 16 & 5,2 \\
\hline 13. Experiencias pasadas & 1 & 3 & 4 & 1,3 \\
\hline 14. Planificación & 2 & 4 & 6 & 1,9 \\
\hline 15. Trabajo de grupo/pares & 1 & 0 & 1 & 0,3 \\
\hline 16. Lista de nombres & 3 & 3 & 6 & 1,9 \\
\hline 17. Capacidad de comprensión & 0 & 2 & 2 & 0,6 \\
\hline
\end{tabular}




\begin{tabular}{|c|c|c|c|c|}
\hline 18. Conocimiento investigado & 34 & 22 & 56 & 18,2 \\
\hline 19. Comprobación de nivel & 0 & 0 & 0 & 0 \\
\hline 20. Habla espontánea & 10 & 4 & 14 & 4,5 \\
\hline 21. Sugerencias y ejemplos & 6 & 20 & 26 & 8,4 \\
\hline Total de interacciones & 178 & 129 & 307 & \\
\hline Porcentaje de interacciones & $57,9 \%$ & $42,1 \%$ & & \\
\hline
\end{tabular}

En cuanto al proceso de transformación que está explícito en categorías de interacción pedagógica, como: sugerencias, ejemplos y decisiones, es palpable tanto en la preparación de guías para la interpretación crítica de texto proporcionados, como en la selección de una didáctica coherente con las características generales de los docentes en formación y su campo disciplinar.

De modo similar, el proceso de nueva comprensión se manifiesta en la autocrítica y el repaso de progresos, lo cual implica el uso de determinadas estrategias de documentación, análisis y socialización de conocimientos. En retrospectiva, el docente 2 contribuyó a un aprendizaje significativo y trascendental en la construcción de un repertorio pedagógico e investigativo de los docentes en formación en el área de la cultura física, recreación y deporte.

\section{DISCUSIÓN}

El estudio pionero en indagar el CPC de docentes con experiencia, mediante categorías que reportan el pensamiento pedagógico, es autoría de Gatboton (1999). Si bien dicha investigación tuvo un diseño de estudio de casos múltiples de nivel descriptivo y comparativo, la diferencia fundamental respecto a la presente investigación es que aquella se ocupó de docentes del área de inglés como segunda lengua (English as Second Language, ESL). Ahora bien, otra similitud con Gatboton $(1999,2008)$, es la identificación de un grupo dominante de pensamientos pedagógicos (p. 40), cuya frecuencia se puede demostrar y estimar a través de un inventario de las acciones que realizan los profesores de manera cotidiana en sus clases.

Cabe aclarar que las llamadas unidades de pensamiento pedagógico (1999), que posteriormente fueron denominadas categorías de pensamiento pedagógico (Mullock,
2006), porque agrupaban a las primeras y eran más amplias y definibles. En realidad, las primeras son indicadores del comportamiento de los docentes, y las segundas son dimensiones de una acción pedagógica exclusiva de los profesores. Todo lo anterior, de acuerdo con Shulman (1987), permite inferir los procesos de razonamiento pedagógico: comprensión, transformación, instrucción, evaluación, reflexión y nueva comprensión.

Ahora bien, los hallazgos de la presente investigación demuestran que el comportamiento del docente, en gran medida, obedece a interacciones pedagógicas bilaterales u horizontales respecto a sus estudiantes. En otros términos, el comportamiento del estudiantado tiene incidencia directa en el comportamiento del profesor y las decisiones que este realiza. Así, los futuros licenciados en cultura física, recreación y deporte aportan al desarrollo de la clase y además condicionan el conocimiento pedagógico del contenido que muestra el docente. Además, los docentes en formación toman la iniciativa en ciertos tipos de interacción, y están en capacidad de aceptar o rechazar las actividades que proponen los docentes expertos.

Por tanto, el presente estudio difiere de visión unilateral y vertical de la acción pedagógica de los docentes con experiencia, en cambio, propone que la interacción pedagógica puede ser básicamente simétrica o asimétrica. Así, la simétrica sucede en los momentos en que los estudiantes asumen la misma conducta del docente, es decir imitan el ejemplo del docente o reproducen las ideas de este. Luego, la asimétrica acontece cuando los estudiantes asumen un comportamiento diferente al del docente, es decir que, actúan conforme a sus convicciones, motivaciones, conocimientos, entre otros, para hacerse notar en clase. 
A partir de los estudios de Gatboton $(1999,2008)$ y Mullock (2006) no es posible reflexionar sobre cómo los docentes con experiencia y los docentes en formación construyen un CPC de la asignatura. Además, los resultados expuestos en el presente apartado emergen de la sistematización y confrontación de los modos de interacción pedagógica de profesores y estudiantes. De esta manera, las diferencias en las interacciones son generadas por las diferencias que los conocimientos, las creencias, las motivaciones y los valores, entre otros, causan en los procesos del razonamiento pedagógico de los actores educativos.

\section{CONCLUSIONES}

A partir de los resultados se plantean algunas conclusiones sobre las semejanzas y diferencias entre las concepciones y opiniones de docentes y estudiantes, en torno a una misma asignatura.

1. La sistematización de los patrones de interacciones pedagógicas, tanto de los docentes expertos como de docentes en formación,

\section{REFERENCIAS BIBLIOGRÁFICAS}

Aldana, G.M. (2012). La formación investigativa: su pertinencia en pregrado. Revista Virtual Universidad Católica del Norte, 35, 367-379.

Álvarez-Sánchez, Y. (2013). La pedagogía socrática como fundamento de la investigación formativa. Revista de la Universidad de la Salle, 60, 241-264.

Anzola, O.L. (2005). La investigación formativa en los procesos de investigación asumidos en la Universidad. Revista Sotavento, 10, 68-73.

De la Ossa, J.; Pérez, A.; Patiño, R. y Montes, D. (2012). La investigación formativa como una necesidad en el pregrado. Revista Colombiana de Ciencia Animal 4(1), 1-3.

Denzin, N.K. y Lincoln, Y.S. (2012). La investigación cualitativa como disciplina y como práctica. En: N. Denzin e Y. Lincoln (coord.). El campo de la investigación cualitativa. Manual de investigación cualitativa. (Vol. I.) (pp. 43-101). Barcelona: Gedisa.

Gatbonton, E. (1999). Investigating Experienced ESL Teachers' Pedagogical Knowledge. The Modern Language Journal 83(1), 35-50. permite elaborar un perfil del razonamiento pedagógico implícito en las acciones y decisiones realizadas en la cotidianidad de las aulas de clase.

2. Los docentes en formación de Licenciatura en Cultura Física, Recreación y Deporte que cursan, en semestres iniciales, las asignaturas de Investigación Formativa I y II, demuestran que tienen un papel activo en el comportamiento y la selección de estrategias de enseñanza de los docentes expertos.

3. La cultura física, la recreación y el deporte como área de formación profesional está a cargo de docentes expertos que poseen un razonamiento pedagógico complejo, que puede ser indagado mediante la genealogía de categorías propuestas por Gatbonton (1999, 2008) y Mullock (2006).

Gatbonton, E. (2008). Looking beyond teachers' classroom behaviour: Novice and experienced ESL teachers' pedagogical knowledge. Language Teaching Research 12(2), 161-182.

Hancock, D.R. y Algozzine, B. (2006). Key terms. Doing Case Study Research (pp. 85-87). Estados Unidos América: Teacher College Press.

Hernández, R.; Fernández,C.y Baptista,P.(2010). Muestreo en la investigación cualitativa. En: Metodología de la investigación (pp. 392-405). 5a. ed. México: McGrawHill.

Lara, G. (2006). Investigación formativa. Una visión integral para profesiones de la salud. Revista Ciencias de la Salud, 4, 161-176.

López, H. (2011). Investigación formativa. Una estrategia en la práctica del cuerpo académico: estudios en educación. Ponencia presentada en el XI Congreso Nacional de Investigación Educativa. México: Universidad Veracruzana.

Ley 30 de 1992. Por la cual se organiza el servicio público de la educación superior. Congreso de la República de Colombia. 
Marczyk, G.; DeMatteo, D. y Festinger, D. (2005) General types of research designs and approaches. En: Essentials of Research Design and Methodology. Hoboken: John Wiley \& Sons.

McMillan, J. y Schumacher, S. (2005) Investigación educativa: una introducción conceptual. 5a. ed. Madrid: Pearson Educación.

Mullock, B. (2006). The Pedagogical Knowledge Base of Four TESOL Teachers. The Modern Language Journal, 90, 48-66.

Sancho Gil, J.M. (2001). Docencia e investigación en la universidad: una profesión, dos mundos. Revista Educar, 28, 41-60.
Shulman, L.S. (1986). Those Who Understand Knowledge Growth in Teaching. S.M. Wilson (ed.) (2004). The Wisdom of Practice: Essays on Teaching, Learning, and Learning to Teach (pp. 187-215). Hoboken: Jossey Bass.

Shulman, L.S. (1987). Knowledge and Teaching: Foundations of the New Reform. En: S.M. Wilson (ed.) (2004). The Wisdom of Practice: Essays on Teaching, Learning, and Learning to Teach (pp. 217-271). Hoboken: Jossey Bass. 